Publ. RIMS Kyoto Univ.

Vol. 6 (1970/71), 483-502

\title{
On the Inverse of Monoidal Transformation
}

\author{
By
}

Shigeo Nakano

\section{Introduction}

When we have a complex analytic manifold $X$ and a complex analytic submanifold $M$ of codimension $r \geqq 2$ of $X$, we can form the monoidal transform $\widetilde{X}$ of $X$ with centre $M$. (By a manifold, we shall understand a paracompact connected one through this paper.) $\widetilde{X}$ is a complex analytic manifold with the same dimension $n$ as $X$, there exists a holomorphic mapping $\pi$ from $\widetilde{X}$ onto $X$, and $\pi$ is an analytic homeomorphism between $X-S$ and $X-M$, where $S=\pi^{-1}(M)$. (More properly, we should say $(\widetilde{X}, \pi)$ is the monoidal transform of $X$.) $S$ is an analytic submanifold of $\widetilde{X}$ of codimension 1 , and is in a peculiar position in $\widetilde{X}$ : The restriction of $\pi$ to $S$ makes $\pi: S \rightarrow M$ an analytic fibre bundle with projective $(r-1)$-space as the standard fibre. (More specifically, $S$ is the normal bundle of $M$ in $X$, with the zero cross section deleted and "divided" by the group $\mathbb{C}^{*}$ operating as multiplication by constants on each fibre.) If we denote the fibre $\pi^{-1}(a)$ by $L_{a}(a \in M)$, then we have $[S]_{L_{a}}=[e]^{-1}$, where $[S]$ and $[e]$ denote the complex line bundles defined by the divisor $S$ of $\widetilde{X}$ and the hyperplane $e$ of $\mathbb{P}^{r-1}=L_{a}$ respectively, and $[S]_{L_{a}}$ denotes the restriction of $[S]$ to $L_{a}$.

Now the inverse problem of the monoidal transformation is the following: Suppose we have a complex analytic manifold $\widetilde{X}^{n}$ and a submanifold $S$ of $\widetilde{X}$ of codimension 1 . Let $S$ have a structure of a holomorphic fibre bundle over an analytic manifold $M^{m}$ with projective $(r-1)$-space as a standard fibre $(m+r=n)$. Then under what condi- 
tion can we find a complex analytic manifold $X^{n}$ and a holomorphic map $\pi: \widetilde{X} \rightarrow X$ so that $X$ contains $M$ as a submanifold and $\widetilde{X}$ is the monoidal transform of $X$ with centre $M$ ? It is clear that the above condition $[S]_{L_{a}}=[e]^{-1}$ is necessary. It was first shown by K. Kodaira that this condition is sufficient in case $M$ is a single point and $\widetilde{X}$ is a projective algebraic manifold (Kodaira [4]). In case $M=$ a point, we also have results by $\mathrm{H}$. Grauert [2] and K. Kodaira [5] etc. For $M$ of higher dimension, P. A. Griffiths [3] gave a sufficient condition that the inverse problem can be solved affirmatively. B. G. Moǔšzon [7] gave a necessary and sufficient condition in case $X$ is compact and $M$ has as many independent meromorphic functions as complex dimension of M. A. Lascu [6] treats this problem in abstract algebraic geometry. In this paper, we shall give a necessary and sufficient condition for the existence of $X$, namely, we shall prove the

Main Theorem. Let $\widetilde{X}$ be a complex analytic manifold of complex dimension $n \geqq 3$ and $S$ an analytic submanifold of $\widetilde{X}$ of codimension 1. Suppose that $S$ has a structure of an analytic fibre bundle over an analytic manifold $M$ with a projective $(r-1)$-space as the standard fibre and that $r>1$. Denote by $L_{a}$ the fibre over $a \in M$ in the bundle $S \rightarrow M$, and by $[e]$ the complex line bundle over $L_{a} \cong \mathbb{P}^{r-1}$ determined by the hyperplane. Then, in order that there exists an n-dimensional analytic manifold $X$ containing $M$ and $a$ holomorphic map $\pi: \widetilde{X} \rightarrow X$ in such a way that $(\widetilde{X}, \pi)$ is the monoidal transform of $X$ with rentre $M$ and $S=\pi^{-1}(M)$, it is necessary and sufficient that the following conditions are satisfied:

(a) for any $a \in M,[S]_{L_{a}}=[e]^{-1}$,

$(\beta)$ each $L_{a}$ has a neighbourhood $V$ (in $\widetilde{X}$ ) such that $[\mathcal{K}]_{V}$ $=(S)_{V}^{b}$, where $\mathcal{K}$ is the canonical bundle of $V$ and $k$ is a nonnegative integer.

To prove the theorem, we make use of a certain type of cohomology varishing theorem and this theorem will be proved making use of a variant of a method due to A. Andreotti and E. Vesentini [1]. 


\section{§1. A Cohomology Vanishing Theorem}

1.1. We shall consider the following situatior: $V$ is an $n$-dimensional complex analytic manifold and there exist a real valued $\mathbb{C}^{\infty}$ function $\Psi$ and a complex line bundle $\mathscr{B}$ on $V$, with the following properties:

(a) $\Psi$ is plurisubharmonic, i. e. for any point $x$ of $V$, we have

$$
\left(\frac{\partial^{2} \Psi}{\partial x^{j} \partial \bar{x}^{k}}\right) \geqq 0 \quad \text { (positive semi-definite), }
$$

where $\left(x^{j}\right)$ is a system of analytic local coordinates around $x$.

(b) For any real value $A$, the set $\{x \mid \Psi(x) \leqq A\}$ is empty or compact.

(c) $\mathscr{B}$ is negative, this mears the following: $\mathscr{B}$ is defined by a system of transition functions $\left\{e_{a \beta}\right\}$ with respect to an open covering $\left\{U_{\alpha}\right\}$ of $V$, and we have a system of positive valued $C^{\infty}$ functions $\left\{a_{\alpha}\right\}$, $a_{\alpha}$ being defined in $U_{\alpha}$ and having the properties

$$
\begin{aligned}
& a_{\alpha} / a_{\beta}=\left|e_{\alpha \beta}\right|^{2} \quad \text { in } \quad U_{\alpha} \cap U_{\beta}, \\
& \left(\frac{\partial^{2} \log a_{\alpha}}{\partial x^{j} \partial \bar{x}^{-k}}\right)<0 \quad \text { at each point of } U_{\alpha} .
\end{aligned}
$$

(d) The canonical bundle of $V$ is equal to $\mathscr{B}^{k}(k \geqq 0)$.

The theorem we want to establish in this section is the following

Theorem 1 . In the above situation, we have $H^{a}\left(V, \mathcal{O}\left(\mathscr{B}^{-\varepsilon}\right)\right)=0$ for $\varepsilon=1,2, \cdots, q=1, \cdots, n-1$.

The proof by the method of Carleman estimate will be given in the following.

1.2. Let us first establish

Proposition 1. The system of functions $\left\{a_{\alpha}\right\}$ can be so chosen that the Kähler metric on $V$ given by

$$
d s^{2}=\sum \frac{\partial^{2} \log a_{\alpha}^{-1}}{\partial x^{j} \partial \bar{x}^{k}} d x^{j} d \bar{x}^{k}
$$


is complete.

Proof. Take a real valued $C^{\infty}$ function $\mu$ of a real variable $\tau$ such that $\mu(\tau) \geqq 0, \mu^{\prime}(\tau) \geqq 0, \mu^{\prime \prime}(\tau) \geqq 0$ and $\int_{0}^{\infty} \sqrt{\mu^{\prime \prime}(\tau)} d \tau=+\infty$, and replace given $a_{\alpha}$ by $a_{\alpha}^{\prime}=e^{(-\mu(\Psi))} a_{\alpha}$. The new system $\left\{a_{\alpha}^{\prime}\right\}$ satisfies the condition (c) too. Define a Kähler metric on $V$ by (1.1) with $a_{\alpha}^{\prime}$ instead of $a_{\alpha}$ on the right hand side.

If $V$ itself is compact, there is nothing to prove. Assume $V$ is not compact, and take a differentiable arc $\gamma: x=x(t)(0 \leqq t<1)$ in $V$ which is not contained in any compact subset of $V$. Set $f(t)=\Psi(\boldsymbol{x}(t))$, then $f\left(t_{\nu}\right) \rightarrow \infty$ for some sequence $\left\{t_{\nu}\right\}$ of values of $t$. We have:

$$
\begin{aligned}
\left(\frac{d s}{d t}\right)^{2} & =\sum_{j, k}\left\{\mu^{\prime}(f(t)) \frac{\partial^{2} \Psi}{\partial x^{j} \partial \bar{x}^{k}}+\mu^{\prime \prime}(f(t)) \frac{\partial \Psi}{\partial x^{j}} \frac{\partial \Psi}{\partial \bar{x}^{k}}\right\} \frac{d x^{j}}{d t} \frac{d \bar{x}^{k}}{d t} \\
& +\sum \frac{\partial^{2} \log a_{\alpha}^{-1}}{\partial x^{j} \partial \bar{x}^{k}} \frac{d x^{j}}{d t} \frac{d \bar{x}^{k}}{d t} \geqq \mu^{\prime \prime}(f(t))\left|\sum_{j} \frac{\partial \Psi}{\partial x^{j}} \frac{d x^{j}}{d t}\right|^{2} \\
& \geqq \frac{1}{4} \mu^{\prime \prime}(f(t))\left|\frac{d f}{d t}\right|^{2} . \quad\left(x^{j}=x^{j}(x(t))\right)
\end{aligned}
$$

Then

$$
\int_{t=0}^{1} d s \geqq \frac{1}{2} \int_{0}^{1} \sqrt{\mid \mu^{\prime \prime}\left(\left.f((t)) \frac{d f}{d t}\right|^{2}\right.}=+\infty .
$$

Therefore the proposition holds if we take $a_{\alpha}^{\prime}$ instead of the original $a_{\alpha}$.

From now, we shall assume that $\left\{a_{\alpha}\right\}$ is chosen so that (1.1) is a complete Kähler metric, and we shall fix this metric through this section.

1. 3. We consider a Hermitian metric $\left\{b_{\alpha}\right\}$ on the fibres of $\mathscr{B}$, that is to say, $b_{\alpha}$ is a positive valued $C^{\infty}$ function on $U_{\alpha}$ and we have

$$
b_{\alpha} / b_{\beta}=\left|e_{\alpha \beta}\right|^{2} \quad \text { in } U_{\alpha} \cap U_{\beta} .
$$

The system $\left\{a_{\alpha}\right\}$ in $\mathbf{1 . 1} \mathbf{1} .2$ is an example of such metric.

We denote by $\mathscr{D}^{p . q}(\mathscr{B})$ the $\boldsymbol{C}$-vector space of all $\mathscr{B}$-valued differential forms of type $(p, q)$ with compact supports. (a $\mathscr{B}$-valued differential form $\varphi$ is a system $\left\{\varphi_{\alpha}\right\}$ of differential forms, $\varphi_{\alpha}$ being defined on $U_{\alpha}$ and satisfying $\varphi_{\alpha}=e_{\alpha \in} \varphi_{\beta}$ in $U_{\alpha} \cap U_{\beta}$. The metric $\left\{b_{\alpha}\right\}$ on the fibres 
defines a Hermitian inner product in $\mathscr{D}^{p, q}(\mathscr{B})$

$$
(\varphi, \psi)=\int_{V} \frac{1}{b_{\alpha}} \varphi_{\alpha \wedge} * \bar{\Psi}_{\alpha} \quad \varphi, \psi \in \mathscr{D}^{p, q}(\mathscr{B})
$$

and makes it a pre-Hilbert space.

With respect to this inner product, the adjoint of the operator $\bar{\partial}$ : $\mathscr{D}^{p, q}(\mathscr{B}) \rightarrow \mathscr{D}^{p, q+1}(\mathscr{B})$, is given by the operator $\vartheta$ :

$$
(\vartheta \varphi)_{\alpha}=-* D^{\prime} * \varphi_{\alpha}
$$

where

$$
\begin{aligned}
& D^{\prime} \varphi_{\alpha}=\partial \varphi_{\alpha}+\rho_{\alpha \wedge \varphi_{\alpha},} \\
& \rho_{\alpha}=-\partial \log b_{\alpha} .
\end{aligned}
$$

$D^{\prime}: \mathscr{D}^{p, q}(\mathscr{B}) \rightarrow \mathscr{D}^{p+1, q}(\mathscr{B})$ is the $(1,0)$-part of the exterior differentiation defined in terms of the connection $\left\{\rho_{\alpha}\right\}$ on $\mathscr{B}$. As usual we define the Laplace-Beltrami operator $\square$ by

$$
\square=\bar{\partial} \vartheta+\vartheta \bar{\partial} \text {. }
$$

We define another norm $\mathrm{n}(\varphi)$ in $\mathscr{D}^{p, q}(\mathscr{B})$ :

$$
\mathrm{n}(\varphi)^{2}=(\varphi, \varphi)+(\bar{\partial} \varphi, \bar{\partial} \varphi)+(\vartheta \varphi, \vartheta \varphi) .
$$

The completions of $\mathscr{D}^{p, q}(\mathscr{B})$ with respect to the norms $(\varphi, \varphi)^{1 / 2}$ and $\mathrm{n}(\varphi)$ are denoted by $\mathcal{L}^{p, q}(\mathscr{B})$ and $W^{p, q}(\mathscr{B})$ respectively.

We shall recall the following propositions:

Proposition 2. $W^{p, q}(\mathscr{B})$ can be considered as the set of elements $\varphi$ of $\mathcal{L}^{p, q}(\mathscr{B})$ for which $\bar{\partial} \varphi \in \mathcal{L}^{p, q+1}(\mathscr{B})$ and $\vartheta \varphi \in \mathcal{L}^{p, q-1}(\mathscr{B})$. Here $\overline{0}$ and $\vartheta$ are taken in the sense of distributions.

For the proof we refer the reader to Andreotti-Vesentini [1]. (Proposition 5 of p. 93 in the paper. One can also find detailed exposition in Vesentini [8].) We shall recall that we make use of the completeness of the metric in the proof. We shall further recall that

$$
(\bar{\partial} \varphi, \bar{\partial} \varphi)+(\vartheta \varphi, \vartheta \varphi) \leqq \sigma(\square \varphi, \square \varphi)+\frac{1}{\sigma}(\varphi, \varphi)
$$

for any $\sigma>0$ and for any $\mathscr{B}$-valued $C^{\infty}$ form $\varphi$ such that each term in 
the formula is defined. This holds because the metric is complete. (Formula (19) of Andreotti-Vesentini [1], p. 93.) The following proposition is an easy consequence of (1.9).

Proposition 3. If $\varphi \in \mathcal{L}^{p, q}(\mathscr{B})$ is of class $C^{\infty}$ and $\square \varphi \in \mathcal{L}^{p, q}(\mathscr{B})$, then $\varphi$ belongs to $W^{p . q}(\mathscr{B})$. In particular, if $\varphi \in \mathcal{L}^{p, q}(\mathscr{B})$ and $\square \varphi=0$, then $\bar{\partial} \varphi=0$ and $\vartheta \varphi=0$.

This is the content of Cor. 6 and Prop. 7 of Andreotti-Vesentini [1] (p. 93), the contents of Theorem 1 (p. 89) and Theorem 21 (p. 94) of the paper are given in the following Propositions:

Proposition 4. If there exists a positive constant $C$ such that

$$
(\bar{\partial} \varphi, \bar{\partial} \varphi)+(\vartheta \varphi, \vartheta \varphi) \geqq C(\varphi, \varphi)
$$

for all $\varphi \in \mathscr{D}^{p, q}(\mathscr{B})$, then for any $\alpha \in \mathcal{L}^{p, q}(\mathscr{B})$, there exists one and only one element $x$ of $W^{p, q}(\mathscr{B})$ satisfying $\square x=\alpha$.

Here $\square x=\alpha$ is to be understocd in the sense of weak solution, i. e., for any $\varphi \in \mathscr{D}^{p, q}(\mathscr{B})$ we have $(\bar{\partial} x, \bar{\partial} \varphi)+(\vartheta x, \vartheta \varphi)=(\alpha, \varphi)$. But since is elliptic, if $\alpha$ is $C^{\infty}$ then $x$ is also $C^{\infty}$ and the equality $\square x=\alpha$ holds in strong sense.

Proposition 5. With the above notations, if $\alpha$ is $C^{\infty}$ with $\bar{\partial} \alpha=0$, then $\beta=\vartheta x$ is the unique $C^{\infty}$ form in $\mathcal{L}^{p, q-1}(\mathscr{B})$ which satisfies $\bar{\partial} \beta=\alpha$ and $\vartheta \beta=0$.

We define Green's operator $G: \mathcal{L}^{p, q} \rightarrow W^{p, q}$ by $x=G \alpha$. It is also seen that

$$
(G \alpha, G \alpha) \leqq C^{-2}(\alpha, \alpha)
$$

1.4. We have introduced the connection $\left\{\rho_{\alpha}\right\}$ combined with the metric $\left\{b_{\alpha}\right\}$. This enables one to define the covariant differentiation $D$ : $\mathscr{D}^{p, q}(\mathscr{B}) \rightarrow \mathscr{D}^{s+1, q}(\mathscr{B}) \oplus \mathscr{D}^{s, q+1}(\mathscr{B})$ by $D=D^{\prime}+\bar{\partial}, D^{\prime}$ being given in (1.4). It is straightforward to verify the relation

$$
\left(D^{2} \varphi\right)_{\alpha}=\Theta \bigwedge \varphi_{\alpha}
$$


where

$$
\Theta=\bar{\partial} \rho_{\alpha}
$$

is a scalar form on $V$ of type (1.1). We set

$$
\chi=\sqrt{-1} \Theta=\sqrt{-1} \partial \bar{\partial} \log b_{\alpha}
$$

and call this the curvature form of the connection $\left\{\rho_{\alpha}\right\}$.

Since the metric of the base manifold $V$ is Kähler, we have the relations

$$
L \delta^{\prime}-\delta^{\prime} L=\sqrt{-1} \bar{\partial}, \quad \bar{\Lambda}-\bar{\partial} \Lambda=-\sqrt{-1} \delta^{\prime} .
$$

(See, for example, A. Weil [9], p. 44. These formulas are proven for scalar forms there, but they can be applied to $\mathscr{B}$-valued forms too, because the operations are defined for those formis and formulas are of local character.) If we take the adjcint of the first formula of (1.14), we have

$$
D^{\prime} \Lambda-\Lambda D^{\prime}=-\sqrt{-1} \vartheta
$$

As (1.11) and (1.12) show, we have $D^{2}=D^{\prime} \bar{\partial}+\bar{\partial} D^{\prime}$. Hence, denoting by $\mathrm{e}(\chi)$ the exterior multiplication by $\chi$, we have

$$
\begin{aligned}
\mathrm{e}(\chi) \Lambda-\Lambda \mathrm{e}(\chi)= & \sqrt{-1}\left\{\left(D^{\prime} \bar{\partial}+\bar{\partial} D^{\prime}\right) \Lambda-\Lambda\left(D^{\prime} \bar{\partial}+\bar{\partial} D^{\prime}\right)\right\} . \\
= & \sqrt{-1}\left\{D^{\prime}(\bar{\partial} \Lambda-\bar{\Lambda})+D^{\prime} \bar{\Lambda}+\bar{\partial}\left(D^{\prime} \Lambda-\Lambda D^{\prime}\right)+\bar{\partial} \Lambda D^{\prime}\right. \\
& \left.-\left(\Lambda D^{\prime}-D^{\prime} \Lambda\right) \bar{\partial}-D^{\prime} \Lambda \bar{\partial}-(\Lambda \bar{\partial}-\bar{\partial} \Lambda) D^{\prime}-\bar{\partial} \Lambda D^{\prime}\right\} \\
= & (\bar{\partial} \vartheta+\vartheta \bar{\partial})-\left(D^{\prime} \delta^{\prime}+\delta^{\prime} D^{\prime}\right),
\end{aligned}
$$

that is to say,

$$
\mathrm{e}(x) \Lambda-\Lambda \mathrm{e}(\chi)=\square-*^{-1} \square *
$$

a formula first due to Calabi and Vesentini.

1.5. Now we turn to the proof of Theorem 1. It is enough to show $H_{K}^{n-q}\left(V, \Omega^{n}\left(\mathcal{B}^{\Xi}\right)\right)=0(1 \leqq q \leqq n-1)$, because of Serre duality theorem. Here $H_{K}$ means the cohomology group with compact supports. By condition (d), this is equivalent to saying $H_{K}^{n-q}\left(V, \mathcal{O}\left(\mathscr{B}^{k+\varepsilon}\right)\right)=0$.

Set $l=k+\varepsilon(>0)$ and apply the considerations in $\mathbf{1 . 3}$ and $\mathbf{1 . 4}$ to 
$\mathscr{B}^{l}$ instead of original $\mathscr{B}$. We take $b_{\alpha}$ to be equal to $a_{\alpha}^{l}$, where $\left\{a_{\alpha}\right\}$ is the system introduced in 1.2. (The meaning of $\left\{b_{\alpha}\right\}$ in (1.2) has now changed according to the change $\mathscr{B} \rightarrow \mathscr{B}^{l}$, but $\left\{a_{\alpha}\right\}$ keeps the original meaning for $\mathscr{B}$.)

$$
\begin{aligned}
\rho_{\alpha} & =\partial \log b_{\alpha}=l\left(\partial \log a_{\alpha}\right)=l \rho_{\alpha}^{(1)} \\
\chi & =\sqrt{-1} \partial \rho_{\alpha}=l\left(\sqrt{-1} \partial \rho_{\alpha}^{(1)}\right)=l \chi^{(1)} .
\end{aligned}
$$

If we write $\chi^{(1)}$ as $\chi^{(1)}=\sqrt{-1} \sum \chi_{j \bar{k}} d x^{j} \wedge d \bar{x}^{k}$, we see $\chi_{j \bar{k}}=\frac{\partial^{2} \log a_{\alpha}}{\partial x^{j} \partial \bar{x}^{k}}=-g_{j \bar{k}}$ according to the choice of the Kähler metric on $V$. Hence $\mathrm{e}\left(\chi^{(1)}\right)=-L$, $\mathrm{e}(\chi)=(-l) L$ and (1.16) takes the form

$$
\square-*^{-1} \square *=l(\Lambda L-L \Lambda) \text {. }
$$

If $\varphi \in \mathscr{D}^{0, n-q}\left(\mathscr{B}^{l}\right)$, then $(\Lambda L-L \Lambda) \varphi=q \varphi$, and

$$
(\bar{\partial} \varphi, \bar{\partial} \varphi)+(\vartheta \varphi, \vartheta \varphi)=(\square \varphi, \varphi)=l q(\varphi, \varphi)+\left(*^{-1} \square * \varphi, \varphi\right) \geqq l q(\varphi, \varphi)
$$

because $\left(*^{-1} \square * \varphi, \varphi\right)=(\square * \varphi, * \varphi) \geqq 0$. This shows that formula (1.10) holds if we choose $C=l q$.

Intermediate Proposition. For $\varphi \in \mathscr{D}^{0, n-q}\left(\mathscr{B}^{l}\right)$ with $\bar{\partial} \varphi=0$, we can find $\psi \in \mathcal{L}^{0, n-q-1}\left(\mathscr{B}^{l}\right)$ which is $C^{\infty}$ and satisfies $\bar{\partial} \psi=0, \vartheta \psi r=0$ and $(\psi, \psi) \leqq \frac{4}{C}(\varphi, \varphi)$, where $C=l q$. We have the same result for any choice of Hermitian metric on the fibre, provided (1.10) holds with the same value of $C$.

Proof. By Propositions 4 and 5, we see that $\psi=\vartheta G \varphi$ satisfies the requirement except $(\psi, \psi) \leqq \frac{4}{C}(\varphi, \varphi)$. Now since $x=G \varphi$ is in $W^{0, q}\left(\mathscr{B}^{l}\right)$, (1.10) holds for $x$ too. Hence

$$
\overline{(\partial x}, \bar{\partial} x)+(\vartheta x, \vartheta x) \geqq C(x, x) .
$$

Apply (1.9) to $x$ with $\sigma=\frac{C}{2}$, then we have

$$
\begin{aligned}
(\bar{\partial} x, \bar{\partial} x)+(\vartheta x, \vartheta x) & \leqq \frac{2}{C}(\varphi, \varphi)+\frac{C}{2}(x, x) \\
& \leqq \frac{2}{C}(\varphi, \varphi)+\frac{1}{2}\{(\bar{\partial} x, \bar{\partial} x)+(\vartheta x, \vartheta x)\}
\end{aligned}
$$

whence 


$$
(\psi, \psi) \leqq(\bar{\partial} x, \overline{\partial x})+(\vartheta x, \vartheta x) \leqq \frac{4}{C}(\varphi, \varphi) .
$$

It remains to show that we can take $\psi$ to lie in $\mathscr{D}^{0, q-1}\left(\mathscr{B}^{l}\right)$, because this result mears that the $\mathscr{B}^{\varepsilon}$-valued Dolbeault cohomology groups with compact support vanish for type $(0, q), q=1,2, \cdots, n-1$.

1.6. In order to achieve the last point, we proceed as follows: We take a real valued $C^{\infty}$ function $\lambda$ of a real variable $t$, satisfying the conditions

$$
\begin{cases}\lambda(t), \quad \lambda^{\prime}(t), \quad \lambda^{\prime \prime}(t) \geqq 0 & \\ \lambda(t)=\lambda^{\prime}(t)=\lambda^{\prime \prime}(t)=0 & \text { for } t>0 \\ \lambda^{\prime}(t)>0 & \text { for } t>0 \\ \lambda^{\prime \prime}(t)>0 & \text { for } 0<t<1 \\ \lambda^{\prime \prime}(t)=0 & \text { for } 1 \leqq t,\end{cases}
$$

and introduce new metrics on the fibres of $\mathscr{B}^{l}$;

$$
b_{\alpha, \nu}=e^{-\nu \lambda(\Psi-A)} a_{\alpha}^{l} \quad(\nu=1,2,3, \cdots) .
$$

Here $A$ is a constant to be determined later. Then the meaning of the inner product changes according as $\nu$ changes. We shall use $(,)_{\nu}$, $\mathcal{L}_{\nu}^{p, q}, W_{\nu}^{p, q}, \vartheta_{\nu}, \square_{\nu} \cdots$, for symbols corresponding to the metric $\left\{b_{\alpha, \nu}\right\}$, while for original metric $a_{\alpha}^{l}=b_{\alpha, 0}$, we shall omit the suffix 0 .

The change of the curvature form $\chi$ is to be noted. We readily see $\chi_{\nu}=\chi-\nu \omega$ where $\omega=\sqrt{-1} \partial \bar{\partial} \lambda(\Psi-A)$. Hence (1.16), (1.18) take the form

$$
\square_{\nu}-*^{-1} \square_{\nu} *=l(\Lambda L-L \Lambda)+\nu(\Lambda \mathrm{e}(\omega)-\mathrm{e}(\omega) \Lambda) .
$$

If we show

$$
((\Lambda \mathrm{e}(\omega)-\mathrm{e}(\omega) \Lambda) \varphi, \varphi) \geqq 0 \quad \text { for } \quad \varphi \in \mathscr{D}^{0, n-q}\left(\mathscr{B}^{l}\right),
$$

we shall have

$$
\left(\square_{\nu} \varphi, \varphi\right)_{\nu} \geqq l q(\varphi, \varphi) \quad \varphi \in \mathscr{D}^{0, n-q}\left(\mathscr{B}^{l}\right) .
$$

As for (1.19), we have, in a small neighbourhood $V$ of a given point, 


$$
\begin{aligned}
\partial \overline{\partial \lambda}(\Psi-A) & =\partial \sum_{k} \lambda^{\prime}(\Psi-A) \frac{\partial \Psi}{\partial \bar{x}^{k}} d \bar{x}^{k} \\
& =\sum_{j, k}\left\{\lambda^{\prime \prime}(\Psi-A) \frac{\partial \Psi}{\partial x^{j}} \frac{\partial \Psi}{\partial \bar{x}^{k}}+\lambda^{\prime}(\Psi-A) \frac{\partial^{2} \Psi}{\partial x^{j} \partial \bar{x}^{k}} d x^{j} \wedge d \bar{x}^{k}\right\} .
\end{aligned}
$$

The quantities in \{\} make up a positive semi-definite matrix. Hence if we take an orthonormal basis $\left\{\theta_{j}\right\}$ of linear differential forms on $U$, we shall have the expressions

$$
\begin{aligned}
& L=\sqrt{-1} \sum \mathrm{e}\left(\theta_{j}\right) \mathrm{e}\left(\bar{\theta}_{j}\right), \\
& e(\omega)=\sqrt{-1} \sum w_{j \bar{k}} \mathrm{e}\left(\theta_{j}\right) \mathrm{e}\left(\theta_{k}\right),
\end{aligned}
$$

where $\left(w_{j \bar{k}}\right) \geqq 0$. We can find a matrix $\left(t_{l j}\right)$ such that $w_{j \bar{k}}=\sum \bar{t}_{l j} t_{l k}$, The adjoint of $\mathrm{e}\left(\theta_{j}\right)$ is denoted by $\mathrm{i}\left(\theta_{j}\right)$ and is given by $-* \mathrm{e}\left(\overline{\theta_{j}}\right) *$. Because $\left\{\theta_{j}\right\}$ is an orthonormal basis, it can be verified that if $A=\left(a_{1}\right.$, $\left.\cdots, a_{r}\right), B=\left(b_{1}, \cdots, b_{s}\right)$ are set of indices, and if $\theta_{A \wedge} \theta_{B}$ derotes $\theta_{a_{1}} \wedge$ $\cdots \wedge \theta_{a_{r}} \wedge \bar{\theta}_{b_{1}} \wedge \cdots \wedge \bar{\theta}_{b_{s}}$, we have

$$
\left\{\begin{array}{l}
\mathrm{i}\left(\theta_{j}\right)\left(\theta_{j \wedge} \theta_{A \wedge} \bar{\theta}_{B}\right)=\theta_{A \wedge} \bar{\theta}_{B} \\
\mathrm{i}\left(\theta_{j}\right)\left(\theta_{A \wedge} \bar{\theta}_{B}\right)=0
\end{array} \quad \text { for } j \notin A,\right.
$$

and similarly for $\mathrm{i}\left(\bar{\theta}_{k}\right)$. From this we conclude the relations

$$
\left\{\begin{array}{l}
\mathrm{e}\left(\theta_{j}\right) \mathrm{i}\left(\theta_{m}\right)+\mathrm{i}\left(\theta_{m}\right) \mathrm{e}\left(\theta_{j}\right)=\delta_{j_{m}} \\
\mathrm{e}\left(\overline{\theta_{k}}\right) \mathrm{i}\left(\overline{\theta_{m}}\right)+\mathrm{i}\left(\overline{\theta_{m}}\right) \mathrm{e}\left(\overline{\theta_{k}}\right)=\delta_{k n} \\
\mathrm{e}\left(\theta_{j}\right) \mathrm{i}\left(\overline{\theta_{k}}\right)+\mathrm{i}\left(\overline{\theta_{k}}\right) \mathrm{e}\left(\theta_{j}\right)=0 \\
\mathrm{e}\left(\overline{\theta_{j}}\right) \mathrm{i}\left(\overline{\theta_{k}}\right)+\mathrm{i}\left(\overline{\theta_{k}}\right) \mathrm{e}\left(\theta_{j}\right)=0 .
\end{array}\right.
$$

Now $\quad \Lambda \mathrm{e}(\omega)-\mathrm{e}(\omega) \Lambda=\sum_{j, k, m} w_{j k}\left\{\mathrm{i}\left(\bar{\theta}_{m}\right) \mathrm{i}\left(\theta_{m}\right) \mathrm{e}\left(\theta_{j}\right) \mathrm{e}\left(\bar{\theta}_{k}\right)-\mathrm{e}\left(\theta_{j}\right) \mathrm{e}\left(\bar{\theta}_{k}\right) \mathrm{i}\left(\bar{\theta}_{m}\right) \mathrm{i}\left(\theta_{m}\right)\right\}$ can be calculated according to (1.22) and gives

$$
\Lambda \mathrm{e}(\omega)-\mathrm{e}(\omega) \Lambda=\sum w_{j k}\left\{\mathrm{i}\left(\bar{\theta}_{j}\right) \mathrm{e}\left(\bar{\theta}_{k}\right)-\mathrm{e}\left(\theta_{j}\right) \mathrm{i}\left(\theta_{k}\right)\right\} .
$$

Hence if $\varphi$ is a $(0, n-q)$-form with support contained in $U$, we have

$$
\begin{aligned}
((\Lambda \mathrm{e}(\omega)-\mathrm{e}(\omega) \Lambda) \varphi, \varphi) & =\left(\sum_{l, j, k} \bar{t}_{l j} \mathrm{i}\left(\overline{\theta_{j}}\right) t_{l k} \mathrm{e}\left(\overline{\theta_{k}}\right) \varphi, \varphi\right) \\
& =\sum_{l}\left(\sum_{k} t_{l k} \mathrm{e}\left(\overline{\theta_{k}}\right) \varphi, \sum_{j} t_{l j} \mathrm{e}\left(\overline{\theta_{j}}\right) \varphi \geqq 0 .\right.
\end{aligned}
$$

This is enough to establish (1.19).

So we have shown (1.20) with the constant $l q$ which is independent 
of $\varphi$ and $\nu$.

1. 7. Now consider $\varphi \in \mathscr{L}^{0, n-q}\left(\mathscr{B}^{l}\right)$ with $\bar{\partial} \varphi=0$. Set $K=\operatorname{supp} \varphi, A$ $=\max _{\boldsymbol{x} \in K} \Psi(\boldsymbol{x})$. We take $b_{\alpha, \nu}$ with this value of $A$, and apply the Intermediate Proposition to this case. Then there exists $\psi_{\nu} \in \mathcal{L}_{\nu}^{0, n-q-1}\left(\mathscr{B}^{l}\right)$ such that $\vartheta_{\nu} \psi_{\nu}=0, \bar{\partial} \psi_{\nu}=\varphi$ and $\left(\psi_{\nu}, \psi_{\nu}\right)_{\nu} \leqq \frac{4}{l q}(\varphi, \varphi)_{\nu}$. Because $b_{\alpha, \nu} \leqq a_{\alpha}^{l}$ and equality holds on the support of $\varphi$,

$$
\frac{4}{l q}(\varphi, \varphi)=\frac{4}{l q}(\varphi, \varphi)_{\nu} \geqq\left(\psi_{\nu}, \psi_{\nu}\right)_{\nu} \geqq\left(\psi_{\nu}, \psi_{\nu}\right),
$$

and $\left\{\psi_{\nu}\right\}_{\nu=1,2, \cdots}$ form a bounded set in $\mathcal{L}^{0, n-q-1}\left(\mathcal{B}^{l}\right)$. Hence, if we choose a suitable subsequeace if necessary, $\psi_{\nu}$ converges to an element $\psi \in \mathcal{L}^{0, n-q-1}\left(\mathscr{B}^{l}\right)$ weakly. We have $(\psi r, \psi) \leqq \frac{4}{l q}(\varphi, \varphi)$ and $\bar{\partial} \psi=\varphi$ in the sense of distribution.

Set $M=\{x \in V \mid \Psi(x)>A+1\}$, then from (1.23) it follows that

$$
\int_{M} a_{\alpha}^{-l} e^{\nu \lambda(\Psi-A)}\left(\psi_{\nu}\right)_{\alpha} \bigwedge * \overline{\left(\psi_{\nu}\right)_{\alpha}} \leqq\left(\psi_{\nu}, \psi_{\nu}\right)_{\nu} \leqq \frac{4}{l q}(\varphi, \varphi) .
$$

On the other hand, there exists a positive constant $c$ such that $\lambda(\Psi$ $-A) \geqq c$ on $M$. Hence the left hand side of (1.24) is not less than $e^{\nu c} \int_{M} a_{\alpha}^{-l}\left(\psi_{\nu}\right)_{\alpha \wedge} * \overline{\left(\psi_{\nu}\right)_{\alpha}}$. This shows that

$$
\int_{M} a_{\alpha}^{-l}\left(\psi_{\nu}\right)_{\alpha \wedge} * \overline{\left(\psi_{\nu}\right)_{\alpha}} \rightarrow 0 \text { for } \quad \nu \rightarrow \infty .
$$

If we take an element $u \in \mathscr{D}^{0, n-q-1}\left(\mathscr{B}^{\prime}\right)$ with support contained in $M$, then it follows from the above that $(\psi, u)=\lim _{\nu \rightarrow \infty}\left(\psi_{\nu}, u\right)=0$. Hence the support of $\psi$ as a distribution is contained in the compact set $V-M$.

Finally, we can apply to $\psi$ the regularization process as given in Andreotti-Vesentini [1], Lemma 12 p. 97, and obtain a form $\psi_{0}$ $\in \mathscr{D}^{0, n-q-1}\left(\mathscr{B}^{l}\right)$ with $\bar{\partial} \psi_{0}=\varphi$. This completes the proof of Theorem 1 .

\section{§. Construction of a Plurisubharmonic Function}

2.1. We consider an $n$-dimensional complex analytic manifold $\widetilde{X}$ and a submanifold $S$ of codimension 1 , and assume that the condition $(\alpha)$ 
in the Main Theorem is satisfied. $S$ is an analytic bundle of projective spaces $\mathbb{P}^{r-1}$ over an analytic manifold $M^{m}$ with $m+r=n$. Take a point $a$ of $M$ and a small coordinate neighbourhood $D$ of $a$. $D$ can be considered as the domain $\left\{\left.\left(\zeta^{1}, \cdots, \zeta^{m}\right) \in \mathbb{C}^{m}\left|\sum\right| \zeta^{j}\right|^{2}<1\right\}$ in $\mathbb{C}^{m}$, the point $a$ corresponding to the origin and the part $\pi^{-1}(D)$ of $S$ which lies over $D$ has the form $\pi^{-1}(D) \cong D \times \boldsymbol{P}^{r-1}$. We take a set of homogeneous coordinates $\left(\eta^{1}, \cdots, \eta^{r}\right)$ on $\mathbb{P}^{r-1}$. Then $\mathbb{P}$ is covered by coordinate neighbourhoods $\left\{U_{\alpha}\right\} ; U_{\alpha}=\left\{\eta^{\alpha} \neq 0\right\}$, and the set of inhomogeneous coordinates $\left\{\xi_{\alpha}^{\gamma}=\eta^{\gamma} / \eta^{\alpha} \mid \gamma=1, \cdots, \hat{\alpha}, \cdots, r\right\}$ is a set of local coordinates in $U_{\alpha}$. (When we make use of the notation $\xi_{\alpha}^{\alpha}$, it is the constant 1.) Because $[S]_{L_{b}}=[e]^{-1}$ for any $b \in D,[S]_{\pi^{-1}(D)}=q^{*}[e]^{-1}$, where $q$ is the canonical projection $D \times \mathbb{P}^{r-1} \rightarrow \mathbb{P}^{r-1}$ and $[e]$ is the complex line bundle on $\mathbb{P}^{r-1}$ determined by a hyperplane.

$[e]$ on $\mathbb{P}^{r-1}$ can be defined with respect to the open covering $\left\{U_{\alpha}\right\}$ by transition functions

$$
\varepsilon_{\alpha \beta}=\eta^{\beta} / \eta^{\alpha}=\xi_{\alpha}^{\beta} \quad \text { in } \quad U_{\alpha} \cap U_{\beta} .
$$

As the metric on the fibre, we take

$$
a_{\alpha}^{(0)}=\sum_{\gamma}\left|\eta^{\gamma}\right|^{2} /\left|\eta^{\alpha}\right|^{2}=\sum_{\gamma}\left|\xi_{\alpha}^{\gamma}\right|^{2} .
$$

The curvature form of this metric is the Kähler form associated to the standard Hodge metric of $\mathbb{P}^{r-1}$.

On $D \times \mathbb{P}$, the bundle $q^{*}[e]$, which we shall now denote by $[e]$, is defined by (2.1) with respect to $\left\{D \times U_{\alpha}\right\}$. We set

$$
\phi(\zeta)=\sum\left|\zeta^{j}\right|^{2}
$$

and take

$$
a_{\alpha}=e^{\phi(\zeta)} a_{\alpha}^{(0)}
$$

as the metric on the fibres. The curvature form of this metric is again positive definite.

2. 2. Local coordinates on $\pi^{-1}(D)$ can be considered as restrictions of local coordinates on $\widetilde{X}$. More precisely, we can choose a finite open covering $\left\{U_{\lambda}^{\prime}\right\}_{\lambda \in A}$ of $\boldsymbol{P}$ which refines $\left\{U_{\alpha}\right\}$, open sets $V_{\lambda}^{\prime}$ on $\widetilde{X}$ such 
that $V_{\lambda}^{\prime} \cap S=D \times U_{\lambda}^{\prime}$, and systems of local coordinates $\left(z_{\lambda}^{1}, \cdots, z_{\lambda}^{m}, x_{\lambda}^{1}, \cdots\right.$, $\left.\hat{x}_{\lambda}^{\tau(\lambda)}, \cdots, x_{\lambda}^{r}, y_{\lambda}\right)$ on $V_{\lambda}^{\prime}$ in the following manner: $\tau$ is a map $\Lambda \rightarrow\{1, \cdots, r\}$ such that $U_{\lambda}^{\prime} \subset U_{\tau(\lambda)}$.

$$
z_{\lambda}^{j}\left|S=\zeta^{j}, \quad x_{\lambda}^{\gamma}\right| S=\xi_{\tau}^{\gamma}(\lambda)
$$

(We set $x_{\lambda}^{\tau(\lambda)} \equiv 1$. Hence (2.6) holds for $\gamma=\tau(\lambda)$ too.)

Furthermore we may assume that the transition function $e_{\lambda_{\mu}}=y_{\lambda} / y_{\mu}$ for $[S]$ satisfies

$$
e_{\lambda \mu} \mid S=\varepsilon_{\lambda \mu}^{-1}
$$

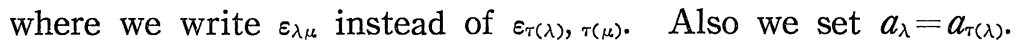

For the moment, we fix the index $j$ and write $z_{\lambda}$ instead of $z_{\lambda}^{j}$. Then $z_{\lambda}-z_{\mu}$ is a holomorphic function on $V_{\lambda}^{\prime} \cap V_{\mu}^{\prime}$ and is zero on $S$. We set

$$
f_{\lambda \mu}=y_{\lambda}^{-1}\left(z_{\lambda}-z_{\mu}\right)
$$

then we have

$$
f_{\lambda \nu}=f_{\lambda \mu}+e_{\lambda \mu}^{-1} f_{\mu \nu} \quad \text { in } \quad V_{\lambda}^{\prime} \cap V_{\mu}^{\prime} \cap V_{\nu}^{\prime} .
$$

In other words $\left\{f_{\lambda \mu}\right\}$ is a 1 -cocycle with values in $\mathcal{O}\left([S]^{-1}\right)$, with respect to the open covering $\mathfrak{B}=\left\{V_{\lambda}^{\prime}\right\}$ of $V^{\prime}=\bigcup_{\lambda} V_{\lambda}^{\prime}$. The restrictions $\varphi_{\lambda \mu}=f_{\lambda \mu} \mid S$ define an element of $Z^{1}(\mathfrak{U}, \mathcal{O}([e]))^{\lambda}$, where $\mathfrak{U}=\left\{D \times U_{\lambda}^{\prime}\right\}$. Since $H^{1}\left(\boldsymbol{P}^{r-1}, \mathcal{O}([e])\right)=0$, we can find holomorphic functions $\varphi_{\lambda}$ on $D \times U_{\lambda}^{\prime}$ such that

$$
\varphi_{\lambda}-\varepsilon_{\lambda \mu} \varphi_{\mu}=\varphi_{\lambda \mu} \quad \text { on } \quad D \times\left(U_{\lambda}^{\prime} \cap U_{\mu}^{\prime}\right)
$$

We extend $\varphi_{\lambda}$ to $U_{\lambda}^{\prime}$ and denote it by $f_{\lambda}$, then $z_{\lambda}^{\prime}=z_{\lambda}-y_{\lambda} f_{\lambda}$ has the property

$$
z_{\lambda}^{\prime}-z_{\mu}^{\prime} \equiv 0\left(\bmod y_{\lambda}^{2}\right) \quad \text { in } V_{\lambda}^{\prime} \cap V_{\mu}^{\prime} \text {. }
$$

We set $f_{\lambda \mu}^{\prime}=y_{\lambda}^{-2}\left(z_{\lambda}^{\prime}-z_{\mu}^{\prime}\right)$ and proceed as before. This time we make use of $H^{1}\left(\boldsymbol{P}^{r-1}, \mathcal{O}\left([e]^{2}\right)\right)=0$, and arrive at $z_{\lambda}^{\prime \prime}=z_{\lambda}^{\prime}-y_{\lambda}^{2} f_{\lambda}^{\prime}$ with $z_{\lambda}^{\prime \prime}-z_{\mu}^{\prime \prime} \equiv 0$ (mod. $\left.y_{\lambda}^{3}\right)$. Since $H^{1}\left(\boldsymbol{P}^{r-1}, \mathcal{O}\left([e]^{k}\right)\right)=0$ for any $k>0$, we can thus proceed as far as we want. Similar procedure can be applied to $x_{\lambda}^{\alpha}$ for 
any fixed $\alpha$ to obtain an approximate holomorphic section of $[S]^{-1}$. Hence,

Proposition 6. Given a positive integer l, we may take local coordinates $\left(z_{\lambda}, x_{\lambda}, y_{\lambda}\right)$ in such a way that the following hold in addition to (2.6) and (2.7).

$$
\left\{\begin{array}{l}
z_{\lambda}^{j}-z_{\mu}^{j}=\left(y_{\lambda}\right)^{l} f_{\lambda \mu}^{j} \\
x_{\lambda}^{\alpha}-e_{\lambda \mu}^{-1} x_{\mu}^{\alpha}=\left(y_{\lambda}\right)^{l} g_{\lambda \mu}^{\alpha}
\end{array} \quad \text { in } \quad V_{\lambda}^{\prime} \cap V_{\mu}^{\prime} .\right.
$$

It may be that we have to replace $V_{\lambda}^{\prime}$ by a smaller open set, in order to secure that $\left(z_{\lambda}^{j}, x_{\lambda}^{\alpha}, y_{\lambda}\right)$ form a local coordinate system. But any way $V^{\prime}=\bigcup_{\lambda} V_{\lambda}^{\prime}$ is an open neighbourhood of $L_{a}$ in $\widetilde{X}$, and it is enough for our purpose.

2. 3. Since $\left\{f_{\lambda \mu}^{j}\right\},\left\{g_{\lambda \mu}^{\alpha}\right\}$ are 1 -cocycles in $Z^{1}\left(\mathfrak{B}, \mathcal{O}\left([S]^{-l}\right)\right), Z^{1}(\mathfrak{B}$, $\mathcal{O}\left([S]^{-l-1}\right)$ ) respectively, it is clear that we can choose $C^{\infty}$ functions $f_{\lambda}^{j}, g_{\lambda}^{\alpha}$ in $V_{\lambda}^{\prime}$ which satisfy

$$
\begin{aligned}
& f_{\lambda}^{j}-e_{\lambda \mu}^{-l} f_{\mu}^{j}=f_{\lambda \mu}^{j}, \\
& g_{\lambda}^{\alpha}-e_{\lambda \mu}^{-l-1} g_{\mu}^{\alpha}=g_{\lambda \mu}^{\alpha} .
\end{aligned}
$$

We set

$$
\left\{\begin{array}{l}
Z^{j}=z_{\lambda}^{j}-\left(y_{\lambda}\right)^{l} f_{\lambda}^{j}=z_{\mu}^{j}-\left(y_{\mu}\right)^{l} f_{\mu}^{j}, \\
X_{\lambda}^{\alpha}=x_{\lambda}^{\alpha}-\left(y_{\lambda}\right)^{l} g_{\lambda}^{\alpha},
\end{array}\right.
$$

then we have

$$
X_{\lambda}^{\alpha}=e_{\lambda \mu}^{-1} X_{\mu}^{\alpha} \quad \text { in } \quad V_{\lambda}^{\prime} \cap V_{\mu}^{\prime} .
$$

Define functions $A_{\lambda}, F$ and $\psi$ by

$$
\begin{aligned}
& \left\{\begin{array}{l}
A_{\lambda}=e_{j}^{\sum_{j}\left|z^{j}\right|^{2}}\left(\sum_{\alpha=1}^{r}\left|X_{\lambda}^{\alpha}\right|^{2}\right) \quad \text { in } V_{\lambda}^{\prime}, \\
F=A_{\lambda} \cdot\left|y_{\lambda}\right|^{2}=A_{\mu} \cdot\left|y_{\mu}\right|^{2},
\end{array}\right. \\
& \psi=\sum_{j}\left|Z^{j}\right|^{2}+F .
\end{aligned}
$$

Since $A_{\lambda}=\left|e_{\lambda \mu}\right|^{-2} A_{\mu}$ by (2.13), $F$ is a well-defined function on $V^{\prime}$. It is clear that $\sum\left|Z^{j}\right|^{2}$ and $A_{\lambda}$ reduce, on $S$, to $\phi=\sum\left|\zeta^{j}\right|^{2}$ and $a_{\lambda}$ respectively.

Proposition 7. Take $l \geqq 3$, then $\psi$ is plurisubharmonic on the 
part of $V^{\prime}$ where $F$ is small enough. More specifically, the Levi form of $\psi$ is not less than a positive definite Hermitian form in $\left(d z^{1} \cdots d z^{m}, d y\right)$ on this part.

Proof. We consider the coordinate neighbourhood $V_{\lambda}^{\prime}$ and verify the assertion in this domain. So we shall omit the suffix $\lambda$ for a moment. For definiteness, let us assume $\tau(\lambda)=r$, hence $\left(z^{1}, \cdots, z^{m}, x^{1}\right.$, $\left.\cdots, x^{r-1}, y\right)$ is the coordinate system, while in the expression $\Sigma\left|X^{\alpha}\right|^{2}$, the summation extends for $\alpha=1,2, \cdots, r$.

Direct computation shows that

$$
\begin{aligned}
& \frac{\partial^{2} \psi^{r}}{\partial z^{j} \partial \bar{z}^{k}}=\delta_{j k}+O(\mid y i), \\
& \frac{\partial^{2} \psi^{r}}{\partial y \partial \bar{y}}=e^{\Sigma\left|z^{\prime}\right|^{2}}\left(1+\sum_{\alpha=1}^{r-1}\left|x^{\alpha}\right|^{2}\right)+O(|y|), \\
& \frac{\partial^{2} \psi}{\partial x^{\alpha} \partial \bar{x}^{\beta}}=e^{\Sigma|z j| 2}|y|^{2}\left(\delta_{\alpha \beta}+O\left(\mid y_{1}^{\prime}\right)\right), \\
& \frac{\partial^{2} y^{r}}{\partial y \partial \bar{x}^{\beta}}=e^{\left.\Sigma\right|_{z} z \mid 2} \bar{y}\left(x^{\beta}+O\left(\mid y_{i}^{\prime}\right)\right), \\
& \frac{\partial^{2} \psi}{\partial y \partial \bar{z} k}=e^{\left.\Sigma|z|\right|^{2}}\left(1+\sum_{\alpha=1}^{r-1}\left|x^{\alpha}\right|^{2}\right)\left\{z^{k} \bar{y}+O\left(|y|^{2}\right)\right\}, \\
& \frac{\partial^{2} \psi^{r}}{\partial z^{j} \partial \bar{x}^{\beta}}=O\left(|y|^{2}\right), \quad \text { and their conjugates. }
\end{aligned}
$$

By taking $V^{\prime}$ smaller if necessary, we may assume that $\sum_{\alpha=1}^{r-1}\left|x_{\alpha}\right|^{2}$ is bounded in $V^{\prime}$, say $\sum\left|x_{\alpha}\right|^{2} \leqq G$. Choose $\eta>0$ such that $\eta G<\frac{1}{3}$, then we have

$$
\begin{aligned}
& \frac{\partial^{2} \psi}{\partial y \partial \bar{y}}(d y, d \bar{y})+\sum_{\beta} \frac{\partial^{2} \psi^{r}}{\partial y \partial \bar{x}^{\beta}}\left(d y, d \bar{x}^{\beta}\right)+\sum_{\alpha} \frac{\partial^{2} \psi}{\partial x^{\alpha} \partial \bar{y}}\left(d x^{\alpha}, d \bar{y}\right) \\
& \quad+\sum_{\alpha, \beta} \frac{\partial^{2} \psi}{\partial x^{\alpha} \partial \bar{x}^{\beta}}\left(d x^{\alpha}, d \bar{x}^{\beta}\right) \\
& =e^{\left.\sum|z|\right|^{\prime 2}} \sum_{\alpha=1}^{r-1}\left|(1+\eta)^{-1 / 2} y d x^{\alpha}+(1+\eta)^{1 / 2} x^{\alpha} d y\right|^{2} \\
& \quad+\left(1-\eta \sum\left|x_{\alpha}\right|^{2}+O(|y|)\right)(d y, d \bar{y})+\sum \bar{y} a_{\beta}\left(d y, d \bar{x}^{\beta}\right) \\
& \left.\left.\quad+\sum y \bar{a}_{\alpha}\left(d x^{\alpha}, d \bar{y}\right)+|y|^{2} \sum \frac{\eta}{1+\eta} \delta_{\alpha \beta}+b_{\alpha \beta}\right)\left(d x^{\alpha}, d \bar{x}^{\beta}\right)\right\}
\end{aligned}
$$




$$
\begin{aligned}
& \geqq e^{\left.\Sigma|z|\right|^{2}}\left\{\frac{2}{3}(d y, d \bar{y})+\sum \bar{y} a_{\beta}\left(d y, d \bar{x}^{\beta}\right)+\sum y \bar{a}_{\beta}\left(d x^{\beta}, d \bar{y}\right)\right. \\
& \left.\quad+|y|^{2} \sum\left(\frac{\eta}{2} \delta_{\alpha \beta}+b_{\alpha \beta}\right)\left(d x^{\alpha} d \bar{x}^{\beta}\right)\right\},
\end{aligned}
$$

where $a_{\alpha}$ and $b_{\alpha \beta}$ are quantities of $O(|y|)$. The last expression is $\geqq 0$ provided $|y|$ is small enough. We can deal with terms containing $\frac{\partial^{2} \psi}{\partial y \partial \bar{z}^{k}}$ etc. similarly, and we see that the Levi form of $\psi$ is equal to the sum of, say $\frac{1}{2}\left(\sum_{j}\left|d z^{j}\right|^{2}+|d y|^{2}\right)$ and a non-negative Hermitian form, if $|y|$ is small enough.

Proposition 8. $\quad\left(-\frac{\partial^{2} \log A_{\lambda}}{\partial x_{\lambda}^{\alpha} \bar{x}_{\lambda}^{\beta}}\right)$ is positive definite for $\left|y_{\lambda}\right|$ small enough.

Proof. This is clear since $A_{\lambda}$ reduces to $a_{\lambda}$ for $y_{\lambda}=0$.

Theorem 2. If $\widetilde{X}, S, M$ satisfy condition ( $\alpha$ ) of the Main Theorem, then, for any $a \in M$, we can find a neighbourhood $V$ of $L_{a}$ in $\widetilde{X}$, such that the conditions $(a),(b),(c)$ in Theorem 1 hold for $V$ and $\mathscr{B}=\left.[S]\right|_{V}$. If we have condition $(\beta)$ in addition, then condition (d) holds too.

Proof. We use the notations in the above. We choose a small positive number $\delta$ such that Propositions 7 and 8 hold for $V=\{\boldsymbol{x}$ $\in \widetilde{X} \mid \psi(\boldsymbol{x})<\delta\}$ and such that $V$ is relatively compact in $V^{\prime}$. If we set $\Psi=(1-\psi / \delta)^{-1}$, then conditions (a) and (b) are satisfied. If we note $\left\{e^{-m \psi} A^{-1}\right\}$ gives a Hermitian metric on the fibres of $[S]$ for any constant $m$, and if we take $m$ big enough, we see that condition (c) is satisfied in view of Propositions 7 and 8 . The last assertion of the theorem is trivial.

Remark. It is important to note that each fibre of $S$ is either contained in $V$ or does not meet $V$. 


\section{§3. The Proof of the Main Theorem}

3.1. First we prove the

Proposition 9. Let $V$ be an $n$-dimensional complex analytic manifold and $S$ a submanifold of $V$ of codimension 1. Suppose that $S$ is analytically homeomorphic to $D \times \mathbb{P}^{r-1}$, where $D$ is a domain in $\mathbb{C}^{m}$ and $m+r=n$, and that $[S]_{s}=[e]^{-1},[e]$ being the complex line bundle on $S$ defined by $D \times$ (hyperplane). If we have $H^{1}(V$, $\left.\mathcal{O}\left([S]^{-\varepsilon}\right)\right)=0$ for $\varepsilon=1,2$, then for any point $a \in D$, there exist a neighbourhood $W$ of $L_{a}$ (=the submanifold of $S$ corresponding to $a \times \mathbb{P})$ in $V$, and a holomorphic map $\pi$ from $W$ to $\Delta=\left\{(z, y) \in \mathbb{C}^{m}\right.$ $\left.\left.\times \mathbb{C}^{r}|| z^{j} \mid<\varepsilon\right\},|y|^{\alpha}<\varepsilon\right\}$ such that $(W, \pi)$ is the monoida! transform of $\Delta$ with centre $\Gamma=$ the linear variety defined by $y^{1}=\cdots=y^{r}=0$. We can idenitify $\Gamma$ with a neighbourhood of $a$ in $D$, and the restriction of $\pi$ to $S$ corresponds to the canonical projection $D \times \mathbb{P} \rightarrow D$ by these identifications.

Proof. Let $\left(\zeta^{1}, \cdots, \zeta^{m}\right)$ be coordinates $\mathbb{C}^{m}$ which contains $D$, and let $\left(\eta^{1}, \cdots, \eta^{r}\right)$ be a set of homogeneous coordinates on $\mathbb{P}^{1} H^{1}(V$, $\left.\mathcal{O}\left([S]^{-1}\right)\right)=0$ implies that the restriction $\Gamma(V, \mathcal{O}) \rightarrow \Gamma\left(S, \mathcal{O}_{S}\right)$ is surjective. Hence there exist holomorphic functions $z^{1}, \cdots, z^{n}$ on $V$, whose restrictions on $S$ are $\zeta^{1}, \cdots, \zeta^{m} \cdot H^{1}\left(V, \mathcal{O}\left([S]^{-2}\right)\right)=0$ implies that $\Gamma\left(V, \mathcal{O}\left([S]^{-1}\right)\right) \stackrel{p}{\rightarrow} \Gamma(S, \mathcal{O}([e]))$ is surjective. The latter contains cross sections corresponding to $\eta^{1}, \cdots, \eta^{r}$. (If we define $[e]$ by transition functions $\varepsilon_{\alpha \beta}=\eta^{\beta} / \eta^{\alpha}$ as in $\S 2$, the cross section corresponding to $\eta^{\gamma}$ is represented by a system of holomorphic functions $\left\{\eta^{\gamma} / \eta^{\alpha}\right\}_{\alpha=1, \cdots, r}$ satisfying $\eta^{\gamma} / \eta^{\alpha}=\varepsilon_{\alpha \beta}\left(\eta^{\gamma} / \eta^{\beta}\right)$.) Hence we can find cross sections $f^{1}, \cdots, f^{r}$ which are mapped to $\eta^{1}, \cdots, \eta^{r}$ by $\rho$.

We may assume we have coordinate neighbourhoods $V_{\lambda}^{\prime}$ in $V$, such that $\bigcup_{\lambda} V_{\lambda}^{\prime} \supset L_{a}, V_{\lambda}^{\prime} \cap S$ has the form $D_{1} \times U_{\lambda}^{\prime}$, and $S$ is defined in $V_{\lambda}^{\prime}$ by a local equation $y_{\lambda}=0$, where $y_{\lambda}$ is a member of a local coordinate system in $V_{\lambda^{\circ}}^{\prime}$ We also assume that (2.5) and (2.8) hold for these data. The cross section $f^{\alpha}$ can be expressed as a system $\left\{f_{\lambda}^{\alpha}\right\}$, where $f_{\lambda}^{\alpha}$ is holomorphic in $V_{\lambda}^{\prime}$ and 


$$
f_{\lambda}^{\alpha}=\left(y_{\lambda} / y_{\mu}\right)^{-1} f_{\mu}^{\alpha} \quad \text { in } \quad V_{\lambda}^{\prime} \wedge V_{\mu}^{\prime} .
$$

Hence we can associate the holomorphic function on $V^{\prime}=\bigcup V_{\lambda}^{\prime}$ which is given by $y_{\lambda} f_{\lambda}^{\alpha}$ in $V_{\lambda^{\circ}}^{\prime}$ We denote this function by $f^{\alpha} \cdot f^{\alpha}$ is in the defining ideal for $S \cap V^{\prime}$. The fact that the map $\rho$ associates $f^{\alpha}$ to $\eta^{\alpha}$ is now expressed by

$$
\left.\left(f^{\alpha} / y^{\lambda}\right)\right|_{s}=\eta^{\alpha} / \eta^{\tau(\lambda)} \text {. }
$$

Now take $\mathbb{C}^{n}$ with linear coordinates $\left(Z^{1}, \cdots, Z^{m}, Y^{1}, \cdots, Y^{r}\right)$ and blow it up with centre $\mathbb{C}^{m}$ defined by $Y^{1}=\cdots=Y^{r}=0$. We denote the monoidal transform by $\widetilde{\mathbb{C}}$, and define an analytic map $\Phi$ from $V^{\prime}$ into $\widetilde{\mathbb{C}}$ by

$$
\Phi(x)=\left(z^{1}(x), \cdots, z^{m}(x), f^{1}(x), \cdots, f^{r}(x)\right) \times\left(f^{1}(x): \cdots: f^{r}(x)\right) .
$$

In view of $(3.1)$, it is seen that $\Phi$ maps $L_{a}$ onto $(0) \times \mathbb{P}^{r-1}$ biholomorphically and the Jacobian of $\Phi$ does not vanish at every point of $L_{a}$. Hence $\Phi$ maps a neighbourhood $W$ of $L_{a}$ onto an open set of $\widetilde{C}$ isomorphically. We can assume that this open set is the transform of a domain $\Delta$ in $\mathbb{C}^{n}$ containing the origin, with centre $\Gamma=\Delta \cap \mathbb{C}^{m}$. The rest of the Proposition is easy to see.

3. 2. The proof of the main theorem is now easy. If we have $\widetilde{X}, S$ and $M$ satisfying conditions $(\alpha)$ and $(\beta)$, then by Theorem 2 , there exists a neighbourhood $V$ of $L_{a}$ for each $a \in M$, satisfying the conditions of Theorern 1 for $\mathscr{B}=[S]_{V}$. Hence we have the conditions of Proposition 9 , and therefore, there exist, for each $a$, a neighbourhood $W_{a}$ of $L_{a}$ in $\widetilde{X}$, a holomorphic map $\pi_{a}$ from $W_{a}$ onto a domain $\Delta_{a}$ in $\mathbb{C}^{n}$ and a linear variety $\Gamma_{a}$ of dimension $m$ in $\Delta_{a}$, such that $\pi_{a}: W_{a} \rightarrow \Delta_{a}$ is the monoidal transform $\Delta_{a}$ with centre $\Gamma_{a}$.

If $W_{a} \cap W_{b} \neq \phi$, we consider the diagram

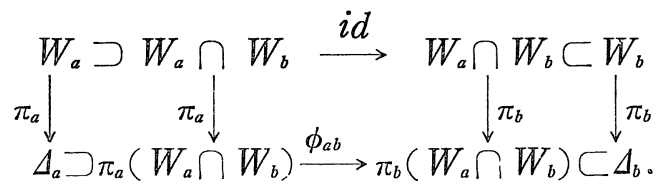

Since $\pi_{a}$ and $\pi_{b}$ are analytic homeomorphism from $W_{a}-S$ to $\Delta_{a}-\Gamma_{a}$ and 
$W_{b}-S$ to $\Delta_{b}-\Gamma_{b}$ respectively, $\phi_{a b}=\pi_{b} \circ i d \circ \pi_{a}^{-1}$ is defined on $\pi_{c}\left(W_{a} \cap W_{b}\right)$ $-\Gamma_{a}$ and maps it isomorphically onto $\pi_{b}\left(W_{a} \cap W_{b}\right)-\Gamma_{b}$. Because $\Gamma_{a}$ is of codimension $r \geqq 2$ in $\Delta_{a}, \phi_{a b}$ can be extended to the whole $\pi_{c}\left(W_{a} \cap W_{b}\right)$ as a holomorphic mapping. Since we can interchange $a$ and $b, \phi_{a b}$ is a biholomorphic homeomorphism from $\pi_{a}\left(W_{a} \cap W_{b}\right)$ onto $\pi_{b}\left(W_{a} \cap W_{b}\right)$.

Thus we can patch together $\Delta_{a}^{\prime}$ s and obtain a possibly non-connected analytic manifold $X_{1}$. In this procedure $\Gamma_{a}^{\prime}$ s are patched together and $\phi_{a b}$ is nothing but the coordinate transfcrmation on $M$, when we identify $\Gamma_{a}$ and $\Gamma_{b}$ with coordicate neighbourhoods arourd $a$ and $b$ respectively. By a standard conrectedness argument, we see that $X_{1}$ is connected and $\Gamma_{a}^{\prime}$ s form a submanifold biholomorphically homeomorphic to $M$. We identify this submanifold with $M$.

The open subsets $\bigcup_{a \in M} W_{a}-S$ of $\widetilde{X}$ and $X_{1}-M$ of $X_{1}$ are biholomorphically homeomorphic with each other, the mapping being given by $\pi_{a}$ on $W_{a}-S$. Hence we can patch together $\widetilde{X}-S$ and $X_{1}$ on these parts, and obtain a manifold $X$. The map $\pi$ from $\widetilde{X}$ to $X$, defined by $\pi=\pi_{a}$ on $W_{a}$ and by $\pi=i d$. on $\widetilde{X}-S$ makes $X$ the monoidal transform of $X$ with centre $M$, and we have $S=\pi^{-1}(M)$. This completes the proof of the sufficiency part of the Main Theorem.

3. 3. The necessity part is trivial. As for condition ( $\beta$ ), we remark that, if $(\widetilde{X}, \pi)$ is the monoidal transform of $X$ with centre $M$ of codimension $r$, then we have $\mathcal{K}_{\widetilde{X}}=\pi^{*} \mathcal{K}_{X} \otimes[S]^{r-1}$, where $\mathcal{K}_{X}, \mathcal{K}_{\widetilde{X}}$ denote the canonical bundles of $X$ and $\widetilde{X}$ respectively.

3. 4. In our Theorem, conditions $(\alpha)$ and $(\beta)$ are certainly necessary and sufficient, but, at the moment, the author doesn't know if these are independert. In fact in the works quoted in the introduction, condition $(\beta)$ is not explicitly mentioned, and $(\beta)$ was used in a technical way in our proof. Hence it may be conjectured that the condition $(\beta)$ will follow from $(\alpha)$ (and with the value $k=r-1$ ). 
Note Added in Proof (March 15, 1971): After this paper was written, Mr. A. Fujiki and the author noticed that condition $(\alpha)$ alone is sufficient to derive the Main Theorem. This supplement will appear in the coming issue of these Publications.

\section{References}

[1] Andreotti, A. and E. Vesentini, Carleman estimates for the Laplace-Beltrami equations on complex manifolds, Publ. Math. I. H. E.S. 25 (1965), 81-155.

[2] Grauert, H., Über Modifikationen und exzeptionelle analytische Mengen, Math. Ann. 146 (1962), 331-368.

[3] Griffiths, P. A., The extension problem in complex analysis II. Amer. J. Math. 88 (1966), 365-446.

[4] Kodaira, K., On Kähler varieties of restricted type. Ann. of Math. 60 (1954), $28-48$.

[5] - On compact analytic surfaces II, Ann. of Math. 78 (1963), 563-626.

[6] Lascu, A., On the contractibility criterion of Castelnuovo-Enriques, Atti Acad. Naz. Lincei, 40 (1966), 1014-1019.

[7] Moǐšzon, B. G., Three papers in Izv. Akad. Nauk SSSR, Ser. Mat. 30 (1966), On $n$-dimensional compact varieties with $n$ algebraically independent meromorphic functions I, II, III, Amer. Math. Transl. Ser. 2. 63 (1967), 51-178.

[8] Vesentini, E., On Levi convexity of complex manifolds and cohomology vanishing theorem, Tata Institute Lecture Notes (1967).

[9] Weil, A., Introduction à l'étude des variétés kähleriennes, Act. Sci. Ind. 1267, Hermann, 1958. 